Report no. $01 / 20$

\title{
Schur complement preconditioning for elliptic systems of partial differential equations
}

\author{
D. Loghin and A. J. Wathen
}

\begin{abstract}
One successful approach in the design of solution methods for saddle-point problems requires the efficient solution of the associated Schur complement problem ([26], [19]). In the case of problems arising from partial differential equations the factorization of the symbol of the operator can often suggest useful approximations for this problem. In this work we examine examples of preconditioners for regular elliptic systems of partial differential equations based on the Schur complement of the symbol of the operator and highlight the possibilities and some of the difficulties one may encounter with this approach.
\end{abstract}

Oxford University Computing Laboratory

Numerical Analysis Group

Wolfson Building

Parks Road

Oxford, England OX1 3QD

November, 2001 


\section{Contents}

1 Introduction 3

2 Definitions and results 4

3 Pseudo-differential operators for preconditioning $\quad 6$

4 Applications $\quad 9$

4.1 Elliptic systems of order one . . . . . . . . . . . . . . . . . . . 9

4.2 Stokes problem . . . . . . . . . . . . . . . . . . 10

4.3 Oseen equations . . . . . . . . . . . . . . . . . . . 11

4.4 2D Magnetostatic equations . . . . . . . . . . . . . . . 12

5 Numerical experiments $\quad 14$

5.1 Elliptic systems of order one . . . . . . . . . . . . . . . . . 15

5.2 Stokes and Oseen equations . . . . . . . . . . . . . . . 16

5.3 2D Magnetostatic problem . . . . . . . . . . . . . . . . . 18

6 Conclusion $\quad 19$ 


\section{Introduction}

Elliptic systems of partial differential equations arise naturally in many practical applications. They generally model physical systems, although there are many examples where they may be associated with a saddle-point problem, where the variables involved do not necessarily take on a physical meaning (e.g., Lagrange multipliers). The systems under consideration here are regular elliptic in the sense of Douglis and Nirenberg [11] and Agmon, Douglis and Nirenberg [1].

Useful guidelines for the analysis of preconditioners for regular elliptic systems were derived by Chen and Strikwerda in [9]. They essentially show that a useful preconditioner can be taken to be a regular elliptic operator with the same order (inside the domain and on the boundary) as the original operator. In particular, they show that the preconditioned system has a condition number which is bounded with respect to the natural norm of the problem. This is a welcome extension of the work of Faber, Manteuffel and Parter [15] and Manteuffel and Parter [25] for scalar elliptic equations.

While [9] provides a useful tool for the analysis of preconditioners for elliptic systems, the issue of design is not always a straightforward matter. In this work, we try to fill this gap by constructing preconditioners based on the Schur complement of the symbol of the matrix operator. Our approach is motivated by results in the field of preconditioning discretizations of saddle-point problems which we outline below.

The discretization of saddle-point problems generally yields indefinite, non-symmetric linear systems of equations of the form

$$
K \mathbf{x}=\left(\begin{array}{cc}
A & B_{1}^{t} \\
B_{2} & C
\end{array}\right)\left(\begin{array}{l}
\mathbf{x}_{u} \\
\mathbf{x}_{p}
\end{array}\right)=\left(\begin{array}{l}
\mathbf{f} \\
\mathbf{q}
\end{array}\right) .
$$

where we assume $A$ is a non-singular matrix; we note here that the variables $\mathbf{x}_{p}$ correspond to the equations of constraint in the problem.

Optimal iterative solution methods for (1.1) have already been devised theoretically: assuming $A$ is invertible, let

$$
S=C-B_{2} A^{-1} B_{1}^{t}
$$

denote the Schur complement of $A$ in $K$. It is shown in [26], [19] that block preconditioners of the form

$$
P_{L}=\left(\begin{array}{cc}
A & 0 \\
B_{1} & S
\end{array}\right), \quad P_{R}=\left(\begin{array}{cc}
A & B_{2}^{t} \\
0 & S
\end{array}\right)
$$

will lead to convergence in at most two iterations. Moreover, if $C=0$, the block-diagonal preconditioner

$$
P_{C}=\left(\begin{array}{cc}
A & 0 \\
0 & S
\end{array}\right)
$$

will lead to convergence in at most four iterations.

When the structure of the matrix $K$ arises from the discretization of a matrix differential operator, it is natural to consider the same ideas for the symbol of this operator (see Section 3). In particular, the Schur complement of the symbol turns out to be a useful concept in the design of efficient preconditioners. However, in general, this operator 
is a pseudo-differential operator the discretization of which is not always straightforward, if possible at all. We examine this issue for the case of several standard elliptic systems of partial-differential equations.

The paper is structured as follows. In section 2 we review some definitions and results concerning regular elliptic systems. In section 3 we outline our approach to preconditioner design. In particular, we show that preconditioners based on the Schur complement of the symbol are elliptic operators of the same order as the original operator which is a necessary condition for optimal preconditioning in the sense of [9]. Section 4 presents various examples of Schur complement preconditioners derived in this fashion. Finally, section 5 presents some selected numerical results.

\section{Definitions and results}

In this section we introduce some notation and definitions concerning regular elliptic systems.

Consider the following general linear system of PDE

$$
\begin{aligned}
& \mathcal{L}(\mathbf{x}, D) \mathbf{u}(\mathbf{x})=\mathbf{f}(\mathbf{x}) \quad \mathbf{x} \in \Omega \subset \mathbb{R}^{n}, \\
& \mathcal{B}(\mathbf{x}, D) \mathbf{u}(\mathbf{x})=\mathbf{g}(\mathbf{x}) \quad \mathbf{x} \in \partial \Omega,
\end{aligned}
$$

where $D=\left(\partial_{1}, \cdots, \partial_{n}\right)$ and $\mathcal{L}(\mathbf{x}, D), \mathcal{B}(\mathbf{x}, D)$ are matrix differential operators with smooth coefficients of size $N \times N$ and $m \times N$, respectively, where $m$ is the order of the system to be specified below.

The following definitions describe regular ellipticity in the sense of Douglis and Nirenberg [11].

Let $\boldsymbol{\xi} \in \mathbb{R}^{n}$; we denote the $l_{2}$-norm of $\boldsymbol{\xi}$ by $|\boldsymbol{\xi}|$. In the following, the deg operator refers to the degree in $\boldsymbol{\xi}$, unless otherwise stated. We denote the $(i, j)$ entry of $\mathcal{L}$ by $\mathcal{L}_{i j}$.

Definition 1 DN ellipticity. A matrix operator $\mathcal{L}(\mathbf{x}, D)$ with real coefficients is said to be elliptic if the following conditions hold:

(i) there exist integers $s_{i} \leq 0$ with $\max _{i} s_{i}=0$ and $t_{j}>0$ such that

$$
\operatorname{deg}\left(\mathcal{L}_{i j}(\mathbf{x}, \boldsymbol{\xi})\right) \leq s_{i}+t_{j}, \quad i, j=1, \ldots, N
$$

(ii) the principal part of $\mathcal{L}$, denoted by $\mathcal{L}^{0}$ and defined as the sum of terms of $\mathcal{L}(\mathbf{x}, \boldsymbol{\xi})$ homogeneous in $\boldsymbol{\xi}$ of degree $s_{i}+t_{j}$, satisfies

$$
\chi(\mathbf{x}, \boldsymbol{\xi})=\operatorname{det}\left(\mathcal{L}^{0}(\mathbf{x}, \boldsymbol{\xi})\right) \neq 0 \quad \forall \mathbf{x} \in \Omega, \boldsymbol{\xi} \in \mathbb{R}^{n} \backslash\{\mathbf{0}\} ;
$$

If $n=2$ we also require

(iii) the polynomial $\chi\left(\mathbf{x}, \boldsymbol{\xi}+z \boldsymbol{\xi}^{\prime}\right)$ in $z \in \mathbb{C}$ has exactly $m$ roots with positive imaginary part for all linearly independent $\boldsymbol{\xi}, \boldsymbol{\xi}^{\prime} \in \mathbb{R}^{2}$.

We say the operator $\mathcal{L}$ has order

$$
m=\frac{1}{2} \operatorname{deg}(\chi(\mathbf{x}, \boldsymbol{\xi}))=\frac{1}{2} \sum_{i} s_{i}+t_{i}>0
$$

and DN numbers $(\mathbf{s}, \mathbf{t})$ where $\mathbf{s}_{i}=s_{i}, \mathbf{t}_{j}=t_{j}, i, j=1, \ldots, N$. 
In the following the principal part of a matrix differential operator is defined as in (ii).

A regular elliptic system $(\mathcal{L}, \mathcal{B})$ requires $\mathcal{L}$ to be uniformly elliptic and $\mathcal{B}$ to satisfy a complementing boundary condition which guarantees the well-posedness of (2.1). This condition is defined below.

Definition 2 Complementing boundary condition. Let $\mathcal{L}$ be an elliptic operator with DN numbers $(\mathbf{s}, \mathbf{t})$. A matrix differential operator $\mathcal{B}$ is said to fulfill the complementing boundary condition for $\mathcal{L}$ if

(i) there exist integers $r_{k}$ such that

$$
\operatorname{deg}\left(\boldsymbol{B}_{k j}(\mathbf{x}, \boldsymbol{\xi})\right) \leq r_{k}+t_{j}, \quad k=1, \ldots, m,
$$

and such that $\boldsymbol{B}_{k j}=0$ for $r_{k}+t_{j}<0$.

(ii) Let $\mathbf{x}_{0} \in \partial \Omega$ and let $\left(\xi_{1}, \ldots, \xi_{n-1}, t\right)$ denote a new coordinate system where $t$ is the coordinate in the direction of the inward normal to $\partial \Omega$ at $\mathbf{x}_{0}$. Let $D_{t}=\left(\xi_{1}, \ldots, \xi_{n-1}, \mathrm{~d} / \mathrm{d} t\right)$. The initial value problem with constant coefficients

$$
\begin{aligned}
& \mathcal{L}^{0}\left(\mathbf{x}_{0}, D_{t}\right) \mathbf{w}(t)=0 \quad t>0, \\
& \mathcal{B}^{0}\left(\mathbf{x}_{0}, D_{t}\right) \mathbf{w}(t)=0 \quad t=0,
\end{aligned}
$$

has a unique solution satisfying $\lim _{t \rightarrow \infty} w(t)=0$, which is the trivial solution. We say the operator $\mathcal{B}$ has DN numbers $(\mathbf{t}, \mathbf{r})$ where $\mathbf{t}$ is as above and $\mathbf{r}_{k}=r_{k}, k=1, \ldots, m$.

Finally, we have

Definition 3 The system $(\mathcal{L}, \mathcal{B})$ is a regular elliptic system of order $m$ with DN numbers $(\mathbf{s}, \mathbf{t}, \mathbf{r})$ if

(i) $\mathcal{L}$ is a uniformly elliptic operator of order $m$ with DN numbers $(\mathbf{s}, \mathbf{t})$.

(ii) $\mathcal{B}$ satisfies the complementing boundary condition for $\mathcal{L}$ and has DN numbers $(\mathbf{t}, \mathbf{r})$.

It is known (see [18], [41]) that a regular elliptic operator $\mathcal{K}=(\mathcal{L}, \mathcal{B}): V \rightarrow W$ is a Fredholm operator for which there holds

(i) $\operatorname{dim} \operatorname{ker}(\mathcal{K})<\infty$;

(ii) $\operatorname{dim} \operatorname{coker}(\mathcal{K})=\operatorname{dim}(W / \operatorname{im}(\mathcal{K}))<\infty$

where $\operatorname{ker}(\mathcal{K}), \operatorname{coker}(\mathcal{K})$ and $\operatorname{im}(\mathcal{K})$ are the kernel, cokernel and the image of $\mathcal{K}$, respectively. The index of a Fredholm operator is

$$
\operatorname{index}(\mathcal{K})=\operatorname{dim} \operatorname{ker}(\mathcal{K})-\operatorname{dim} \operatorname{coker}(\mathcal{K})
$$

It is shown in [9] that a useful preconditioner for an invertible regular elliptic operator $\mathcal{K}$ is another invertible regular elliptic operator with the same index and DN numbers as $\mathcal{K}$. We include this result below.

Let $\mathcal{K}: V \rightarrow W$ be a regular elliptic operator with DN numbers $(\mathbf{s}, \mathbf{t}, \mathbf{r})$, where

$$
\begin{aligned}
V & =H^{l+\mathbf{t}}(\Omega) \\
W & =H^{l-\mathbf{s}}(\Omega) \times H^{l-\mathbf{r}-1 / 2}(\partial \Omega)
\end{aligned}
$$


with norms given by

$$
\begin{gathered}
\|\mathbf{v}\| V^{2}=\sum_{i=1}^{N}\left\|\mathbf{v}_{i}\right\| H^{l+\mathbf{t}_{i}}(\Omega)^{2} \\
\|\mathbf{w}\| W^{2}=\sum_{i=1}^{N}\left\|\mathbf{w}_{i}\right\| H^{l-\mathbf{s}_{i}}(\Omega)^{2}+\sum_{i=1}^{N}\left\|\mathbf{w}_{i}\right\| H^{l-\mathbf{r}_{i}-1 / 2}(\partial \Omega)^{2}
\end{gathered}
$$

Let

$$
\|\mathbf{v}\| V / \operatorname{ker}(\mathcal{K})=\inf \left\{\left\|\mathbf{v}^{\prime}\right\| V: \mathbf{v}^{\prime}-\mathbf{v} \in \operatorname{ker}(\mathcal{K}), \mathbf{v}, \mathbf{v}^{\prime} \in V\right\}
$$

The following result can be found in [9].

Theorem 1 Let $\mathcal{K}: V / \operatorname{ker}(\mathcal{K}) \rightarrow \operatorname{im}(\mathcal{K})$ be a regular elliptic operator with $D N$ numbers $(\mathbf{s}, \mathbf{t}, \mathbf{r})$. If the regular elliptic operator $\mathcal{P}$ is invertible as a map from $V / \operatorname{ker}(\mathcal{K})$ to $\operatorname{im}(\mathcal{K})$ and has the same $D N$ numbers and index as $\mathcal{K}$ then for some $\gamma<\infty$

$$
\begin{gathered}
\left\|\mathcal{P}^{-1} \mathcal{K}\right\| V / \operatorname{ker}(\mathcal{K})<\gamma, \quad\left\|\mathcal{K}^{-1} \mathcal{P}\right\| V / \operatorname{ker}(\mathcal{K})<\gamma \\
\left\|\mathcal{K}^{-1}\right\| W<\gamma, \quad\left\|\mathcal{P}^{-1}\right\| W<\gamma
\end{gathered}
$$

\section{Pseudo-differential operators for preconditioning}

While Theorem 1 provides a tool for the analysis of preconditioners for regular elliptic systems, the question of finding a suitable preconditioner is still to be addressed. We do so here, by deriving a Schur complement preconditioner based on the symbol of our operator. Since our preconditioner is in general a pseudo-differential operator, we recall first some useful definitions and results concerning pseudo-differential operators (see for example [14], [18], [38]) Let

$$
\hat{\phi}(\boldsymbol{\xi})=\mathcal{F} \phi(\mathbf{x})=(2 \pi)^{-n / 2} \int_{\mathbb{R}^{n}} \phi(\mathbf{x}) e^{i \mathbf{x} \cdot \boldsymbol{\xi}} \mathrm{d} \mathbf{x}
$$

denote the Fourier transform of a function $\phi \in C^{\infty}\left(\mathbb{R}^{n}\right)$ and let $\mathcal{F}^{-1}$ denote the inverse Fourier transform.

Definition 4 A pseudo-differential operator $A: C^{\infty}\left(\mathbb{R}^{n}\right)$ is defined by

$$
A \phi=\mathcal{F}^{-1}(A(\boldsymbol{\xi}) \hat{\phi}(\boldsymbol{\xi})) .
$$

The function $A(\boldsymbol{\xi})$ is called the symbol of $A$.

Note that if $A(\boldsymbol{\xi})$ is a polynomial, then, by the above definition, $A(D)$ is a linear differential operator in the classical sense.

Let now $A(\boldsymbol{\xi})$ be a measurable function such that $\left|D^{\boldsymbol{\alpha}} A(\boldsymbol{\xi})\right| \leq C_{\boldsymbol{\alpha}}(1+|\boldsymbol{\xi}|)^{m-|\boldsymbol{\alpha}|}$. The class of such functions will be denoted by $S^{m}$.

Definition 5 A pseudo-differential operator with symbol $A(\boldsymbol{\xi})$ is said to have order $m$ if $A(\boldsymbol{\xi}) \in S^{m}$. 
It follows immediately that if $A_{i}(\boldsymbol{\xi}) \in S^{m_{i}}, i=1,2$, then $A_{1}(\boldsymbol{\xi}) A_{2}(\boldsymbol{\xi}) \in S^{m_{1}+m_{2}}$. Just as in the polynomial case, the symbol $A(\boldsymbol{\xi})$ can be written as the sum of a principal homogeneous part $A^{0}(\boldsymbol{\xi}) \in S^{m}$ of degree $m$ (i.e., $A^{0}(t \boldsymbol{\xi})=t^{m} A^{0}(\boldsymbol{\xi})$ ) and a remainder of lower order $A(\boldsymbol{\xi})-A^{0}(\boldsymbol{\xi}) \in S^{m-\varepsilon}, \varepsilon>0$.

Remark 3.1 In the following we extend the use of the deg operator to denote more generally the order of a symbol $A(\boldsymbol{\xi})$.

Definition 6 A pseudo-differential operator with symbol $A(\boldsymbol{\xi}) \in S^{m}$ is elliptic if its principal homogeneous part $A^{0}(\boldsymbol{\xi})$ satisfies

$$
A^{0}(\boldsymbol{\xi}) \neq 0, \quad \forall \boldsymbol{\xi} \neq \mathbf{0} .
$$

A result which we will use later is that if $A_{i}(\boldsymbol{\xi})$ have order $m_{i}, i=1,2$ and $A_{2}(\boldsymbol{\xi})$ is elliptic, then $A_{1}(\boldsymbol{\xi}) / A_{2}(\boldsymbol{\xi}) \in S^{m_{1}-m_{2}}$.

Remark 3.2 The notion of DN-ellipticity extends naturally to pseudo-differential operators. Thus, the degree is replaced by the order of the operator in condition (i) of Definition 1 while the principal part of a matrix of pseudo-differential operators will be the sum of principal homogeneous parts of individual entries which satisfy condition (ii) of the same definition.

Let now $\mathcal{L}(D)$ be a matrix differential operator with constant coefficients and with symbol $\mathcal{L}(\boldsymbol{\xi})$ partitioned so that the equations corresponding to the variables of constraint come last in the system (cf. (1.1)):

$$
\mathcal{L}(\boldsymbol{\xi})=\left(\begin{array}{cc}
A(\boldsymbol{\xi}) & B_{1}^{t}(\boldsymbol{\xi}) \\
B_{2}(\boldsymbol{\xi}) & C(\boldsymbol{\xi})
\end{array}\right)
$$

where $A$ is such that $\operatorname{det}(A(\boldsymbol{\xi})) \neq 0, \forall \boldsymbol{\xi} \in \mathbb{R}^{n}$. This condition guarantees that the following decompositions of $\mathcal{L}(\boldsymbol{\xi})$ make sense

$\mathcal{L}(\boldsymbol{\xi})=\left(\begin{array}{cc}A(\boldsymbol{\xi}) & 0 \\ B_{2}(\boldsymbol{\xi}) & S(\boldsymbol{\xi})\end{array}\right)\left(\begin{array}{cc}I & A(\boldsymbol{\xi})^{-1} B_{1}^{t}(\boldsymbol{\xi}) \\ 0 & I\end{array}\right)=\left(\begin{array}{cc}I & 0 \\ B_{2}(\boldsymbol{\xi}) A(\boldsymbol{\xi})^{-1} & I\end{array}\right)\left(\begin{array}{cc}A(\boldsymbol{\xi}) & B_{1}^{t}(\boldsymbol{\xi}) \\ 0 & S(\boldsymbol{\xi})\end{array}\right)$

where

$$
S(\boldsymbol{\xi})=C(\boldsymbol{\xi})-B_{2}(\boldsymbol{\xi}) A(\boldsymbol{\xi})^{-1} B_{1}^{t}(\boldsymbol{\xi})
$$

Thus, we are led to consider the preconditioners (cf. (1.3), (1.4))

$\mathbf{P}_{L}(D)=\left(\begin{array}{cc}A(D) & 0 \\ B_{2}(D) & S(D)\end{array}\right), \mathbf{P}_{C}(D)=\left(\begin{array}{cc}A(D) & 0 \\ 0 & S(D)\end{array}\right), \mathbf{P}_{R}(D)=\left(\begin{array}{cc}A(D) & B_{1}^{t}(D) \\ 0 & S(D)\end{array}\right)$

where $S(D)$ is a pseudo-differential operator with symbol $S(\boldsymbol{\xi})$ given by (3.1).

The above choice of preconditioners is further motivated by Theorem 1 and the following result. 
Lemma 2 Let $\mathcal{L}(D)$ be a $N \times N$ elliptic matrix differential operator with $D N$ numbers $(\mathbf{s}, \mathbf{t})$ and assume that

$$
\mathcal{L}(D)=\left(\begin{array}{cc}
A(D) & B_{1}^{t}(D) \\
B_{2}(D) & C(D)
\end{array}\right)
$$

where $A(D)$ be an elliptic operator such that

$$
\operatorname{deg} \operatorname{det}[A(\boldsymbol{\xi})]_{k l}=\operatorname{deg} \operatorname{det}\left[A^{0}(\boldsymbol{\xi})\right]_{k l}, \quad \forall k, l,
$$

where $[A(\boldsymbol{\xi})]_{k l}$ denotes the $(k l)$-cofactor of $A(\boldsymbol{\xi})$. Let $S(D)$ be a pseudo-differential operator defined by

$$
S(D) \phi(\mathbf{x})=(2 \pi)^{-n / 2} \int_{\mathbb{R}^{n}}\left(C(\boldsymbol{\xi})-B_{2}(\boldsymbol{\xi}) A(\boldsymbol{\xi})^{-1} B_{1}^{t}(\boldsymbol{\xi})\right) \hat{\phi}(\boldsymbol{\xi}) e^{-i \boldsymbol{\xi} \cdot \mathbf{x}} \mathrm{d} \boldsymbol{\xi} .
$$

Then $\mathbf{P} \in\left\{\mathbf{P}_{L}, \mathbf{P}_{C}, \mathbf{P}_{R}\right\}$ is an elliptic operator with DN numbers $(\mathbf{s}, \mathbf{t})$.

Proof We have to verify the three requirements of Definition 1 . We first show that $\operatorname{deg} \mathbf{P}(\mathbf{x}, \boldsymbol{\xi}) \leq$ $\mathbf{t}_{i}+\mathbf{s}_{j}, i, j,=1, \ldots, N$. This is true for all the $\mathbf{P}_{i j}$ except for the entries corresponding to $S(D)$. Since $C(\boldsymbol{\xi})$ also satisfies the above condition, we only need to show that $\operatorname{deg}\left(B_{2}(\boldsymbol{\xi}) A(\boldsymbol{\xi})^{-1} B_{1}^{t}(\boldsymbol{\xi})\right)_{i j} \leq$ $\mathbf{s}_{i}+\mathbf{t}_{j}$. First note that

$$
\begin{aligned}
\operatorname{deg}\left(A(\boldsymbol{\xi})^{-1}\right)_{k l} & =\operatorname{deg} \operatorname{det}[A(\boldsymbol{\xi})]_{k l} / \operatorname{det} A(\boldsymbol{\xi}) \\
& =\operatorname{deg} \operatorname{det}\left[A^{0}(\boldsymbol{\xi})\right]_{k l}-\operatorname{deg} \operatorname{det} A^{0}(\boldsymbol{\xi}) \\
& =\sum_{\mathbf{s}_{q} \neq \mathbf{s}_{l}, \mathbf{t}_{q} \neq \mathbf{t}_{k}}\left(\mathbf{s}_{q}+\mathbf{t}_{q}\right)-\sum_{q}\left(\mathbf{s}_{q}+\mathbf{t}_{q}\right) \\
& =-\left(\mathbf{s}_{l}+\mathbf{t}_{k}\right)
\end{aligned}
$$

where we used the ellipticity of $A$ and homogeneity of $A^{0}$. Hence

$$
\begin{aligned}
\operatorname{deg}\left(B_{2}(\boldsymbol{\xi}) A(\boldsymbol{\xi})^{-1} B_{1}^{t}(\boldsymbol{\xi})\right)_{i j} & =\operatorname{deg}\left(B_{2}(\boldsymbol{\xi})\right)_{i k}+\operatorname{deg}\left(B_{1}^{t}(\boldsymbol{\xi})\right)_{l j}+\operatorname{deg}\left(A(\boldsymbol{\xi})^{-1}\right)_{k l} \\
& \leq\left(\mathbf{s}_{i}+\mathbf{t}_{k}\right)+\left(\mathbf{s}_{l}+\mathbf{t}_{j}\right)-\left(\mathbf{s}_{l}+\mathbf{t}_{k}\right) \\
& =\mathbf{s}_{i}+\mathbf{t}_{j}
\end{aligned}
$$

and requirement (i) follows. On the other hand, if $\operatorname{deg} S_{i j}(\boldsymbol{\xi})=\mathbf{s}_{i}+t_{j}$ for some $i, j$, then

$$
\operatorname{deg} S_{i j}(\boldsymbol{\xi})=\operatorname{deg}\left(C_{i j}^{0}(\boldsymbol{\xi})-\left(B_{1}^{0}(\boldsymbol{\xi})\left(A^{0}(\boldsymbol{\xi})\right)^{-1}\left(B_{2}^{0}(\boldsymbol{\xi})\right)^{t}\right)_{i j}\right) ;
$$

Thus, the principal part of $S$ is $S^{0}(\boldsymbol{\xi})=C^{0}(\boldsymbol{\xi})-B_{2}^{0}(\boldsymbol{\xi})\left(A^{0}(\boldsymbol{\xi})\right)^{-1}\left(B_{1}^{0}(\boldsymbol{\xi})\right)^{t}$. Hence, $\operatorname{det} \mathbf{P}^{0}(\boldsymbol{\xi})=$ $\operatorname{det} \mathcal{L}^{0}(\boldsymbol{\xi})$ and requirements (ii) and (iii) are satisfied since $\mathcal{L}$ is elliptic. Thus, by definition, $\mathbf{P}(D)$ is elliptic with DN numbers $(\mathbf{s}, \mathbf{t})$.

Remark 3.3 Note that requirement (3.2) holds for all the applications we consider in the next section. In particular, it holds for the class of problems for which $A(\boldsymbol{\xi})=p(\boldsymbol{\xi}) I$, i.e., $A(\boldsymbol{\xi})$ is a diagonal operator. 
Remark 3.4 If $C=0$ and $A(\boldsymbol{\xi})=Q(\boldsymbol{\xi}) I$, the above integral representation of $S(D)$ simplifies to the one given below

$$
S(D) \phi=B_{2}(D) B_{1}^{t}(D)\left(\mathcal{G}_{Q} * \phi\right)
$$

for suitable functions $\phi$, where $\mathcal{G}_{Q}$ denotes the fundamental solution for the operator $Q(D)$, and the * operator denotes the usual convolution of two functions.

To define completely our preconditioners, we need suitable boundary conditions. We follow the guidelines in [9] and define the following preconditioners for the elliptic system $\mathcal{K}=(\mathcal{L}, \mathcal{B})$

$$
\mathcal{P}_{R}=\left(\mathbf{P}_{R}, \mathcal{B}_{R}\right), \quad \mathcal{P}_{C}=\left(\mathbf{P}_{C}, \mathcal{B}_{C}\right), \quad \mathcal{P}_{L}=\left(\mathbf{P}_{L}, \mathcal{B}_{L}\right),
$$

where $\mathcal{B}_{R}, \mathcal{B}_{C}, \mathcal{B}_{L}$ are chosen so that they have the same DN numbers as $\mathcal{B}$.

Remark 3.5 If $\mathcal{P} \in\left\{\mathcal{P}_{R}, \mathcal{P}_{C}, \mathcal{P}_{L}\right\}$ satisfies the complementing boundary condition of Definition 2 and has the same index as $\mathcal{K}$, then it is an optimal preconditioner for $\mathcal{K}$ in the sense of Theorem 1.

Remark 3.6 While the above requirements for optimality can be easily checked for our preconditioners, the numerical solution may not accommodate our pseudo-differential operator or its boundary conditions so readily. Indeed, we may find that either the discretization of $S(D)$ or the implementation of the boundary conditions are not in general straightforward.

\section{Applications}

In this section we consider several examples of regular elliptic systems and derive corresponding Schur complement preconditioners based on the Schur complement of the symbol. While any of the preconditioners $\left\{\mathcal{P}_{L}, \mathcal{P}_{C}, \mathcal{P}_{R}\right\}$ is a candidate for an efficient preconditioner in the sense of Theorem 1, we consider without loss of generality only the case $\mathcal{P}=\mathcal{P}_{R}$, even for symmetric problems.

\subsection{Elliptic systems of order one}

Consider Darcy's equations for groundwater flow in a domain $\Omega \subset \mathbb{R}^{n}$ with boundary $\Gamma=\Gamma_{D} \cup \Gamma_{N}$

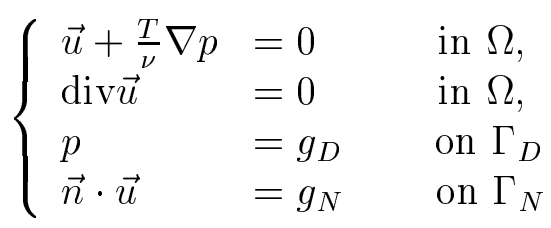

where $T$ is the permeability tensor, $\nu$ is the dynamic viscosity, $p$ is the residual pressure and $\vec{u}$ is the Darcy velocity. 
We note here that problem (4.1) is equivalent to the scalar second-order elliptic equation

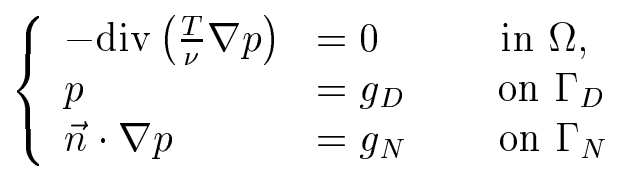

We can write (4.1) in the general form $(2.1)$ where $\mathbf{u}=(\vec{u}, p), N=n+1$ and the operator $\mathcal{K}=(\mathcal{L}, \mathcal{B})$ is given by

$$
\mathcal{L}(D)=\left(\begin{array}{cc}
\nu T^{-1} & D^{t} \\
D & 0
\end{array}\right), \quad \mathcal{B}(\mathbf{x}, D)=\left(\vec{n}(\mathbf{x}) \delta\left(\Gamma_{N}\right), \delta\left(\Gamma_{D}\right)\right)
$$

Moreover, it is straightforward to check that $\mathcal{K}$ is a regular elliptic operator of order $m=$ 1 with DN numbers $\mathbf{s}=(-1,-1,0), \mathbf{t}=(1,1,2)$ if $n=2$ and $\mathbf{s}=(-1,-1,-1,0), \mathbf{t}=$ $(1,1,1,2)$ if $n=3$. Since $m=1$, there is only one DN number $r$ given by $r=-1$ on $\Gamma_{N}$ and $r=-2$ on $\Gamma_{D}$ for $n=2,3$. It is well-known that system (4.1) has a unique solution with no constraints on the data so that $\operatorname{index}(\mathcal{K})=0$.

The symbol of $\mathcal{L}$ is

$$
\mathcal{L}(\boldsymbol{\xi})=\left(\begin{array}{cc}
\nu T^{-1} & \boldsymbol{\xi}^{t} \\
\boldsymbol{\xi} & 0
\end{array}\right)
$$

and the Schur complement in this case is approximated by the pseudo-differential operator with symbol $S(\boldsymbol{\xi})=-\nu^{-1} \boldsymbol{\xi} T \boldsymbol{\xi}^{t}$. Since this is a polynomial, $S(D)$ is a differential operator, which turns out to be the operator of problem (4.2). A suitable preconditioner in this case is therefore the preconditioner $\mathcal{P}=\left(\mathbf{P}_{R}, \mathcal{B}_{R}\right)$ given by

$$
\mathbf{P}_{R}=\left(\begin{array}{cc}
\nu T^{-1} & D^{t} \\
0 & -\nu^{-1} \operatorname{div} T \nabla
\end{array}\right), \quad \mathcal{B}_{R}=\left\{\begin{array}{cl}
(0,0,1) & \text { on } \Gamma_{D} \\
(0,0, \vec{n} \cdot \nabla) & \text { on } \Gamma_{N}
\end{array}\right.
$$

We note here that $\boldsymbol{B}_{R}$ has the same DN number as $\mathcal{B}$ and was chosen so that index $(\mathcal{P})=$ 0 .

\subsection{Stokes problem}

Consider the Stokes equations in $\Omega \subset \mathbb{R}^{n}$



As before, $\mathbf{u}=(\vec{u}, p), N=n+1$ and the operator $\mathcal{K}=(\mathcal{L}, \mathcal{B})$ is given by

$$
\mathcal{L}(D)=\left(\begin{array}{cc}
-\nu \Delta I_{n} & D^{t} \\
D & 0
\end{array}\right), \quad \mathcal{B}(\mathbf{x}, D)=\left(\begin{array}{ll}
I_{n} & 0
\end{array}\right)
$$

This time $\mathcal{K}$ is a regular elliptic operator of order $m=n$ with DN numbers $\mathbf{s}=$ $(0,0,-1), \mathbf{t}=(2,2,1), \mathbf{r}=(-2,-2)$ if $n=2$ and $\mathbf{s}=(0,0,0,-1), \mathbf{t}=(2,2,2,1), \mathbf{r}=$ 
$(-2,-2,-2)$ if $n=3$. As described in [9], the index of $\mathcal{K}$ is zero, since $\operatorname{dim} \operatorname{ker}(\mathcal{K})=1$, as $p$ is defined up to a constant, and $\operatorname{dim} \operatorname{coker}(\mathcal{K})=1$ since there is one constraint on $\operatorname{im}(\mathcal{K})$, namely, $\int_{\Gamma} \vec{n} \cdot \vec{g} \mathrm{~d} \Gamma=0$.

The symbol of $\mathcal{L}$ is

$$
\mathcal{L}(\boldsymbol{\xi})=\left(\begin{array}{cc}
-\nu|\boldsymbol{\xi}|^{2} I_{n} & \boldsymbol{\xi}^{t} \\
\boldsymbol{\xi} & 0
\end{array}\right)
$$

and the Schur complement in this case has symbol $S(\boldsymbol{\xi})=\nu^{-1}$. Thus $S(D)$ is a scaled identity operator. As before, we choose the preconditioner

$$
\mathbf{P}_{R}=\left(\begin{array}{cc}
-\nu \Delta I_{n} & D^{T} \\
0 & -\nu^{-1}
\end{array}\right), \quad \mathcal{B}_{R}=\mathcal{B}
$$

it is shown in [9] that this operator has the same DN numbers and index as $\mathcal{K}$.

Remark 4.1 A saddle point formulation of the linear elasticity equations (see e.g., [6]) uses a very similar underlying operator with symbol

$$
\mathcal{L}(\boldsymbol{\xi})=\left(\begin{array}{cc}
-\nu|\boldsymbol{\xi}|^{2} I_{n} & \boldsymbol{\xi}^{t} \\
\boldsymbol{\xi} & -\rho
\end{array}\right)
$$

In this case also $S(D)$ is a scaled identity operator with no boundary conditions and symbol $S(\boldsymbol{\xi})=\nu^{-1}-\rho$.

\subsection{Oseen equations}

Consider the Oseen equations with a source term in $\Omega \subset \mathbb{R}^{n}$ with Dirichlet boundary conditions



where $\vec{b} \in \mathbb{R}^{n}, c \in \mathbb{R}$ are assumed to be constant. This time the operator $\mathcal{K}=(\mathcal{L}, \mathcal{B})$ is given by

$$
\mathcal{L}(D)=\left(\begin{array}{cc}
p(D) I_{n} & D^{t} \\
D & 0
\end{array}\right), \quad \mathcal{B}(\mathbf{x}, D)=\left(\begin{array}{ll}
I_{n} & \mathbf{0}
\end{array}\right)
$$

where $p(D)=-\nu \Delta+\vec{b} \cdot \nabla+c$ and the DN numbers and the index are the same as for the Stokes equations.

The symbol of $\mathcal{L}$ is

$$
\mathcal{L}(\boldsymbol{\xi})=\left(\begin{array}{cc}
p(\boldsymbol{\xi}) I_{n} & \boldsymbol{\xi}^{t} \\
\boldsymbol{\xi} & 0
\end{array}\right)
$$

where $p(\boldsymbol{\xi})=-\nu|\boldsymbol{\xi}|^{2}+\vec{b} \cdot \boldsymbol{\xi}+c$; the Schur complement in this case has symbol

$$
S(\boldsymbol{\xi})=-\frac{|\boldsymbol{\xi}|^{2}}{p(\boldsymbol{\xi})}
$$


and thus $S(D)$ is a pseudo-differential operator given by (cf. Remark 3.4)

$$
S(D) \phi=-\Delta\left(\mathcal{G}_{p} * \phi\right),
$$

where $\mathcal{G}_{p}$ is the fundamental solution for the advection-diffusion-reaction operator

$$
p(D)=-\nu \Delta+\vec{b} \cdot \nabla+c .
$$

Thus, a useful preconditioner is given by

$$
\mathbf{P}_{R}=\left(\begin{array}{cc}
p(D) I_{n} & D^{t} \\
0 & S(D)
\end{array}\right)
$$

with boundary operator $\mathcal{B}_{R}=\mathcal{B}$ (see also [9]).

\subsection{D Magnetostatic equations}

Consider solving the 2D magnetostatic problem

$$
\left\{\begin{array}{lll}
\vec{B}-\mu \vec{H} & =0 & \text { in } \Omega, \\
\operatorname{div} \vec{B} & =0 & \text { in } \Omega, \\
\operatorname{rot} \vec{H} & =j & \text { in } \Omega, \\
\vec{n} \cdot \vec{B} & =g_{B} & \text { on } \Gamma_{B}, \\
\vec{t} \cdot \vec{H} & =g_{H} & \text { on } \Gamma_{H}
\end{array}\right.
$$

where $\vec{B}$ is the magnetic flux, $\vec{H}$ is the magnetic field strength and $\mu=\mu_{0} \mu_{r}$ is the permeability tensor (which reduces to a scalar in linear isotropic media), with $\mu_{0}$ the permeability of vacuum and $\mu_{r}$ the relative permeability; $j$ is the imposed current density.

One possible approach to solving (4.8) is to use a saddle-point formulation which minimizes the error in the first equation, while treating all the other equations as constraints. This leads to the following weak formulation (see [29]): find $(\vec{B}, \vec{H}, \lambda, \phi) \in$ $\mathcal{V}^{1} \times \mathcal{V}^{2} \times \mathcal{Q}^{1} \times \mathcal{Q}^{2}$ such that

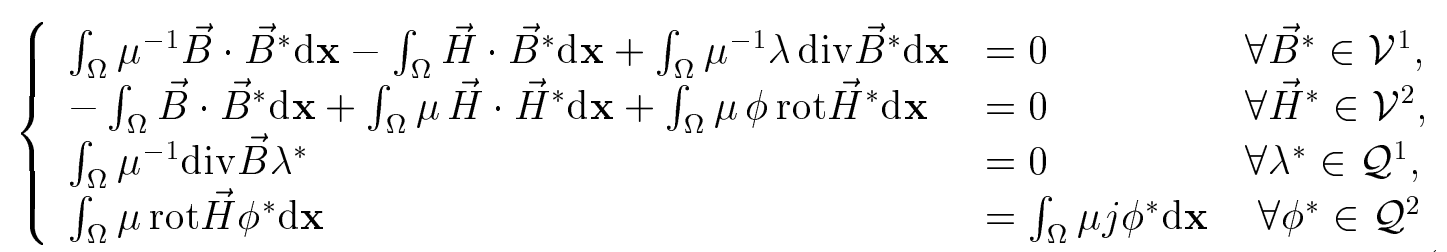

where $\mathcal{Q}^{1}=\mathcal{Q}^{2}=L^{2}(\Omega), \lambda, \phi$ are Lagrange multipliers associated with the constraints $\operatorname{div} \vec{B}=0, \operatorname{rot} \vec{H}=j$, while the boundary constraints are imposed via the choice of spaces

$$
\begin{aligned}
& \mathcal{V}^{1}=H_{\Gamma_{B}}(\operatorname{div}, \Omega)=\left\{\vec{v} \in H(\operatorname{div} ; \Omega):\left.\vec{n} \cdot \vec{v}\right|_{\Gamma_{B}}=0\right\} \\
& \mathcal{V}^{2}=H_{\Gamma_{H}}(\operatorname{rot} ; \Omega)=\left\{\vec{v} \in H(\operatorname{rot} ; \Omega):\left.\vec{t} \cdot \vec{v}\right|_{\Gamma_{H}}=0\right\}
\end{aligned}
$$


where $H(\operatorname{div} ; \Omega), H(\operatorname{rot} ; \Omega)$ are the spaces of square-integrable vector functions with square-integrable div and rot, respectively.

We associate with the saddle-point formulation (4.9) the following regular elliptic system of PDE

$$
\left\{\begin{array}{lll}
\mu^{-1} \vec{B}-\vec{H}-\mu^{-1} \nabla \lambda & =0 & \text { in } \Omega, \\
-\vec{B}+\mu \vec{H}-\mu \operatorname{rot} \phi & =0 & \text { in } \Omega, \\
\mu^{-1} \operatorname{div} \vec{B} & =0 & \text { in } \Omega, \\
\mu \operatorname{rot} \vec{H} & =\mu j & \text { in } \Omega,
\end{array}\right.
$$

with boundary conditions

$$
\begin{cases}\vec{n} \cdot \vec{B}=g_{B} & \text { on } \Gamma_{B} \\ \vec{t} \cdot \vec{H}=g_{H} & \text { on } \Gamma_{H} \\ \phi=0 & \text { on } \Gamma_{B} \\ \lambda=0 & \text { on } \Gamma_{H}\end{cases}
$$

Note that upon elimination of $\vec{B}, \vec{H}$ we find that $\lambda$ and $\phi$ need to satisfy the CauchyRiemann equations with suitable boundary conditions. The above choice of boundary conditions is obtained by requiring system (4.10), (4.11) (i) to be regular and elliptic and (ii) to have the same weak formulation as the saddle-point problem (4.9). We note here that the index of the operator is zero.

The symbol of the operator of equation (4.10) is then given by

$$
\mathcal{L}(\boldsymbol{\xi})=\left(\begin{array}{cc}
A(\boldsymbol{\xi}) & -B^{t}(\boldsymbol{\xi}) \\
B(\boldsymbol{\xi}) & 0
\end{array}\right)
$$

with

$$
A(\boldsymbol{\xi})=\left(\begin{array}{cc}
\mu^{-1} I_{2} & -I_{2} \\
-I_{2} & \mu I_{2}
\end{array}\right), \quad B(\boldsymbol{\xi})=\left(\begin{array}{cc}
\mu^{-1} \boldsymbol{\xi} & \\
& \mu \boldsymbol{\xi}_{\perp}
\end{array}\right)
$$

where $\boldsymbol{\xi}_{\perp}=\left(-\xi_{2}, \xi_{1}\right)$. The boundary operator of (4.11) has symbol given by

$$
\mathcal{B}(\mathbf{x}, \boldsymbol{\xi})=\left(\begin{array}{cccccc}
\vec{n}_{1} \delta\left(\Gamma_{B}\right) & \vec{n}_{2} \delta\left(\Gamma_{B}\right) & 0 & 0 & \delta\left(\Gamma_{H}\right) & 0 \\
0 & 0 & \vec{t}_{1} \delta\left(\Gamma_{H}\right) & \vec{t}_{2} \delta\left(\Gamma_{H}\right) & 0 & \delta\left(\Gamma_{B}\right)
\end{array}\right) .
$$

The operator is regular elliptic of degree 2 with DN numbers

$$
\mathbf{s}=(-1,-1,-1,-1,0,0), \quad \mathbf{t}=(1,1,1,1,2,2),
$$

with $\mathbf{r}=(-1,-2)$ on $\Gamma_{B}$ and $\mathbf{r}=(-2,-1)$ on $\Gamma_{H}$.

Since $A(\boldsymbol{\xi})$ is singular, the Schur complement is not defined. However, if we consider the perturbed operator $\mathcal{L}_{\epsilon}(\boldsymbol{\xi})$ with $A(\boldsymbol{\xi})$ replaced by $A(\boldsymbol{\xi})+\epsilon I_{4}$, we find the symbol of the Schur complement to be for the case $\nu=1$

$$
S(\boldsymbol{\xi})=\frac{\epsilon(\epsilon+2)}{1+\epsilon}|\boldsymbol{\xi}|^{2} I_{2}
$$

and therefore $S(D)$ is a diagonal scaled $2 \times 2$ matrix Laplacian. 
Remark 4.2 When $\mu \neq 1, I_{2}$ is replaced by a diagonal matrix which is not a multiple of the identity. However, the original problem can be scaled so that $\mu$ is eliminated in the beginning. This is also advisable in practical applications [31].

The resulting preconditioner has the form

$$
\mathbf{P}_{R}=\left(\begin{array}{cc}
I_{4} & -B^{t}(D) \\
0 & S(D)
\end{array}\right)
$$

while a boundary operator which ensures ellipticity for the same DN numbers and the same index is

$$
\mathcal{B}_{R}(\boldsymbol{\xi})=\left(\begin{array}{cccccc}
0 & 0 & 0 & 0 & \delta\left(\Gamma_{H}\right)+\vec{n} \cdot \boldsymbol{\xi} \delta\left(\Gamma_{B}\right) & 0 \\
0 & 0 & 0 & 0 & 0 & \delta\left(\Gamma_{B}\right)+\vec{n} \cdot \boldsymbol{\xi} \delta\left(\Gamma_{H}\right)
\end{array}\right)
$$

\section{$5 \quad$ Numerical experiments}

In this section we present some numerical results which illustrate the performance of preconditioners based on the ideas in this paper. We only consider finite element discretizations here; in particular, we used mixed formulations of the finite element method (see [35], [6] for a general exposition). This approach leads to the following abstract mixed variational formulation

$$
\begin{aligned}
& \text { Find }(u, p) \in V \times Q \subset \mathcal{V} \times \mathcal{Q} \text { such that } \\
& \qquad \begin{aligned}
a(u, v)+b_{1}(v, p) & =f(v) \quad \forall v \in V, \\
b_{2}(u, q)+c(p, q) & =g(q) \quad \forall q \in Q
\end{aligned}
\end{aligned}
$$

where $a(\cdot, \cdot): V \times V \rightarrow \mathbb{R}, b_{i}(\cdot, \cdot): V \times Q \rightarrow \mathbb{R}, c(\cdot, \cdot): Q \times Q \rightarrow \mathbb{R}$ are continuous bilinear forms, $f(\cdot), g(\cdot)$ are continuous linear forms and $V, Q$ are two finite element spaces which satisfy the Babuska-Brezzi (BB) condition. $\mathcal{V}, \mathcal{Q}$ are two Hilbert spaces natural to the problem. This condition guarantees the stability and well-posedness of our discretization (for details see Brezzi and Fortin [6]).

In the examples below, the finite element spaces $V, Q$ will be BB-stable. Moreover, the domain $\Omega$ will be replaced with a set $\mathcal{T}^{h}$ of simplices $T$ of size $\operatorname{diam} T=h_{T}$ with $\max _{T} h_{T}=h$. We will denote by $P_{k}(T)$ the space of polynomials of degree $k$ on $T$, and by $R T_{k}(T), T R_{k}(T)$ the Raviart-Thomas and rotated Raviart-Thomas spaces of degree $k$ (see [32] for details). Since we seek a solution $(u, p)$ as a linear combination of compactlysupported basis functions of $V \times Q$, the resulting linear system has a sparse coefficient matrix with a block structure as in (1.1). We use right-preconditioned GMRES for all problems; a tolerance of $10^{-6}$ for the relative residual is the stopping criterion for all experiments. We implement the preconditioners of Section 4; in each case we describe the construction of the Schur complement as a discrete operator on the 'constraint' space $Q$. 


\subsection{Elliptic systems of order one}

The numerical solution of Darcy's equations (4.1) or equivalently of the mixed formulation for second-order elliptic problems (4.2) has received considerable attention from an approximation point of view [3], [4], [34], [32], [33], [27], [8], [28], as well as from a computational one [5], [10], [17], [36], [39], [2] . We consider here two approaches, primal and dual, which lead to different choices for the spaces $V$ and $Q$ [35, pp 577-588]:

(i) primal approach: $\mathcal{V}=\left(L^{2}(\Omega)\right)^{n}, \mathcal{Q}=H_{\Gamma_{D}}^{1}(\Omega)$ with finite element spaces

$V=\left\{\vec{v} \in\left(L^{2}(\Omega)\right)^{n}: \forall T \in \mathcal{T}^{h},\left.\vec{v}\right|_{T} \in\left(P_{k}(T)\right)^{n}\right\}, Q=\left\{q \in H_{\Gamma_{D}}^{1}(\Omega): \forall T \in \mathcal{T}^{h},\left.q\right|_{T} \in P_{k+1}(T)\right\}$

(ii) dual approach: $\mathcal{V}=H_{\Gamma_{N}}(\operatorname{div} ; \Omega), \mathcal{Q}=L^{2}(\Omega)$ with finite element spaces

$V=\left\{\vec{v} \in H_{\Gamma_{N}}(\operatorname{div} ; \Omega): \forall T \in \mathcal{T}^{h},\left.\vec{v}\right|_{T} \in R T_{k}(T)\right\}, Q=\left\{q \in L^{2}(\Omega): \forall T \in \mathcal{T}^{h},\left.q\right|_{T} \in P_{k}(T)\right\}$

In each case we used the lowest possible order, given by $k=0$.

The bilinear forms for problem (4.1) are

$$
a(\vec{w}, \vec{v})=\int_{\Omega} \nu \vec{w} \cdot T^{-1} \cdot \vec{v} \mathrm{~d} \mathbf{x}, \quad b(\vec{v}, q)=\int_{\Omega} q \operatorname{div} \vec{v} \mathrm{~d} \mathbf{x}, \quad c(r, q)=0 .
$$

We used preconditioner (4.3) for both primal and dual approaches. The Schur complement is a Laplacian with mixed boundary conditions (the operator in (4.2)) which is assembled on the space $Q$. While this assembly process is straightforward for the primal approach, it is not immediate for the dual approach since $Q$ is the space of piecewise constant polynomials on $\mathcal{T}^{h}$ which cannot be used to project a second order operator on. To circumvent this problem, we defined a 'dual' set of vertices which consists of the midpoints of each simplex. The Schur complement was assembled as a standard fivepoint difference operator on this set of vertices. To implement the boundary conditions we used a fictitious boundary, which comprised the vertices corresponding to a strip of fictitious simplices which we added to $\mathcal{T}^{h}$ around the boundary of the domain.

The first test problem considers the case $\nu^{-1} T=I_{n}$. The number of GMRES iterations for the primal approach is displayed in Table 1 for the cases $\Gamma_{N}=\emptyset$ and $\Gamma_{N} \neq \emptyset$. The corresponding results for the dual approach are shown in Table 2. Note that for the primal approach we get convergence in 2 iterations. The reason for this phenomenon is that for this simple test problem, the assembled Schur complement is the Schur complement of the problem up to a constant and the results from [26], [19] apply. However, this does not hold for the dual approach and thus the iteration count is somewhat higher, though still mesh-independent. The above type of preconditioner has already been

\begin{tabular}{c|ccccc}
$h=$ & $1 / 16$ & $1 / 32$ & $1 / 64$ & $1 / 128$ & $1 / 256$ \\
\hline$\Gamma_{N}=\emptyset$ & 2 & 2 & 2 & 2 & 2 \\
$\Gamma_{N} \neq \emptyset$ & 2 & 2 & 2 & 2 & 2
\end{tabular}

Table 1: GMRES iteration counts for the primal approach. 


\begin{tabular}{c|ccccc}
$h=$ & $1 / 16$ & $1 / 32$ & $1 / 64$ & $1 / 128$ & $1 / 256$ \\
\hline$\Gamma_{N}=\emptyset$ & 14 & 15 & 15 & 16 & 16 \\
$\Gamma_{N} \neq \emptyset$ & 14 & 15 & 15 & 16 & 16
\end{tabular}

Table 2: GMRES iteration counts for the dual approach.

\begin{tabular}{r|ccccc}
$h=$ & $1 / 16$ & $1 / 32$ & $1 / 64$ & $1 / 128$ & $1 / 256$ \\
\hline$\Gamma_{N}=\emptyset$ & 18 & 18 & 18 & 18 & 18 \\
$\Gamma_{N} \neq \emptyset$ & 18 & 18 & 18 & 18 & 18
\end{tabular}

Table 3: GMRES iteration counts for the primal approach.

considered in [36] for the dual approach and for Dirichlet boundary conditions only; we refer the reader to this work for an analysis of the preconditioned iterative technique and the numerical results. However, we include here the results for the primal approach using preconditioner (4.3) for the test problem suggested in [36] which takes $T$ to be

$$
T(x, y)=\left(\begin{array}{cc}
1+4\left(x^{2}+y^{2}\right) & 3 x y \\
3 x y & 1+11\left(x^{2}+y^{2}\right)
\end{array}\right) .
$$

The results are included in Table 3. Note that the number of iterations is again constant.

\subsection{Stokes and Oseen equations}

The natural spaces $\mathcal{V}, \mathcal{Q}$ for the solution of the Stokes/Oseen equations are

$$
\mathcal{V}=\left(H_{\Gamma}^{1}(\Omega)\right)^{n}=\left\{\vec{v} \in\left(H^{1}(\Omega)\right)^{n}:\left.\vec{v}\right|_{\Gamma}=0\right\}, \quad \mathcal{Q}=L^{2}(\Omega) / \mathbb{R}
$$

and we consider the following BB-stable finite element spaces

$$
V=\left\{\vec{v} \in \mathcal{V}: \forall T \in \mathcal{T}^{h},\left.\vec{v}\right|_{T} \in\left(P_{k}(T)\right)^{n}\right\}, \quad Q=\left\{q \in \mathcal{Q}: \forall T \in \mathcal{T}^{h},\left.q\right|_{T} \in P_{k-1}(T)\right\}
$$

for the choice $k=2$, which gives rise to the so-called P2P1 discretization. The bilinear forms in (5.1) are given below

$$
a(\vec{w}, \vec{v})=\int_{\Omega}(\nu \nabla \vec{w} \cdot \nabla \vec{v}+(\vec{b} \cdot \nabla) \vec{w} \cdot \vec{v}+c \vec{w} \cdot \vec{v}) \mathrm{d} \mathbf{x}, \quad b(\vec{v}, q)=\int_{\Omega} q \operatorname{div} \vec{v} \mathrm{~d} \mathbf{x}, \quad c(r, q)=0 .
$$

Preconditioning techniques for the Stokes problem (4.4) and the related Oseen problem (4.6) have also received a lot of attention. Preconditioners of type (4.5) for the Stokes problem have been considered in [7], [40], [37], [16], [9] and we will not include either analysis or numerics here (see also [22] for performance of preconditioners equivalent to our Schur complement preconditioner for elasticity problems). We will concentrate instead on the Oseen problem.

While the Stokes preconditioner (4.5) can be shown to be optimal in the sense of ([9]) also for the Oseen operator, numerical experiments in [12] show that for small $\nu$, the 
number of iterations is constant with respect to $h$, but too large for practical purposes. Our Schur preconditioners (4.7) reduce this dependence on the viscosity parameter considerably, while preserving the mesh-independence.

Of all our examples, the Oseen problem is the only one that led to a preconditioner which is a proper pseudodifferential operator. The important issue of discretizing a pseudodifferential operator becomes central to the implementation of our preconditioner. While a finite element discretization of a pseudodifferential operator can be devised via a straightforward though costly discretization of the integral representation (3.3) (see [14]), the issue of efficiency cannot be abandoned at this stage. Recall that the Schur complement for the Oseen problem is given by

$$
S(D) \phi=-\Delta\left(\mathcal{G}_{\Theta} * \phi\right)
$$

where $\mathcal{G}_{\Theta}$ is the fundamental solution for the advection-diffusion-reaction operator $\Theta(D)=$ $-\nu \Delta+\vec{b} \cdot \nabla+c$. First note that the quantity we are actually interested in is the inverse of $S(D)$ given by

$$
S^{-1}(D) \phi=(-\nu \Delta+\vec{b} \cdot \nabla+c)\left(\mathcal{G}_{\Delta} * \phi\right)=-\nu \phi+(\vec{b} \cdot \nabla+c)\left(\mathcal{G}_{\Delta} * \phi\right) .
$$

This is the operator we project onto our finite element space in order to approximate the action of the inverse of $S(D)$. Computationally, this only requires the approximation of the convolution $\mathcal{G}_{\Delta} * \phi$ onto our bounded domain, which we achieve by solving a Laplacian problem with periodic boundary conditions. More precisely, we project

$$
-\Delta \psi=\phi
$$

onto a subspace of $H^{1}(\Omega)$ of periodic functions and then project

$$
S^{-1} \phi=-\nu \phi+(\vec{b} \cdot \nabla+c) \psi
$$

onto $H^{1}(\Omega)$. Note that [20] (see also [24], [21]) suggest empirically a discretization of (5.2) with homogeneous Neumann boundary conditions which is a more practical choice of boundary conditions and which leads to performance comparable to that presented in this work for the case of periodic boundary conditions.

We also remark here that preconditioner (4.7) was considered first in [20], [23]; more results and analysis can be found in [13], [24]. In particular, it is shown in [24] that the eigenvalues of the preconditioned discrete operator are bounded independently of $h$ for both time-dependent and time-independent problems and for both stable and stabilized discretizations.

The test problem we consider is the so-called regularized driven cavity problem which we solve using a Picard iteration (see [21]) for details. The average number of GMRES iterations over the number of Picard steps is displayed in Table 4 for a range of viscosity parameters and mesh sizes. Again, the number of iterations seems to be independent of the mesh-size, as noticed for the Darcy problem. 


\begin{tabular}{r|cccccccc}
$\nu=$ & $1 / 10$ & $1 / 20$ & $1 / 40$ & $1 / 80$ & $1 / 160$ & $1 / 320$ & $1 / 640$ & $1 / 1280$ \\
\hline$h=1 / 16$ & 3.00 & 4.37 & 5.85 & 7.22 & 10.66 & 15.54 & 19.54 & 27.38 \\
$1 / 32$ & 3.14 & 4.42 & 5.85 & 7.85 & 10.88 & 15.55 & 22.27 & 30.30 \\
$1 / 64$ & 2.83 & 4.33 & 6.16 & 7.85 & 11.12 & 16.11 & 22.22 & 30.62
\end{tabular}

Table 4: GMRES iteration counts for the steady-state $(c=0)$ driven cavity problem.

\subsection{D Magnetostatic problem}

Our final example is the 2D magnetostatic problem (4.8) which we solve using the saddlepoint formulation (4.9). The spaces of interest are now $\mathcal{V}=\mathcal{V}^{1} \times \mathcal{V}^{2}, \mathcal{Q}=\mathcal{Q}^{1} \times \mathcal{Q}^{2}$ with $\mathcal{V}^{1}, \mathcal{V}^{2}, \mathcal{Q}^{1}, \mathcal{Q}^{2}$ described in Section 4.4. The finite element spaces we employ are given by

$$
\begin{aligned}
& V^{1}=\left\{\vec{B} \in H_{\Gamma_{B}}(\operatorname{div} ; \Omega): \forall T \in \mathcal{T}^{h},\left.\vec{B}\right|_{T} \in R T_{k}(T)\right\}, Q^{1}=\left\{\lambda \in L^{2}(\Omega): \forall T \in \mathcal{T}^{h},\left.\lambda\right|_{T} \in P_{k}(T)\right\}, \\
& V^{2}=\left\{\vec{H} \in H_{\Gamma_{H}}(\operatorname{div} ; \Omega): \forall T \in \mathcal{T}^{h},\left.\vec{H}\right|_{T} \in T R_{k}(T)\right\}, Q^{2}=\left\{\phi \in L^{2}(\Omega): \forall T \in \mathcal{T}^{h},\left.\lambda\right|_{T} \in P_{k}(T)\right\},
\end{aligned}
$$

while the bilinear forms are

$a(\vec{u}, \vec{v})=\int_{\Omega} \mu^{-1}(\vec{B}-\mu \vec{H}) \cdot\left(\vec{B}^{*}-\mu \vec{H}^{*}\right) \mathrm{d} \mathbf{x}, \quad b(\vec{u}, \vec{p})=\int_{\Omega} \mu^{-1} \operatorname{div} \vec{B} \lambda \mathrm{d} \mathbf{x}+\int_{\Omega} \mu \operatorname{rot} \vec{H} \phi \mathrm{d} \mathbf{x}$

where $\vec{u}=(\vec{B}, \vec{H})^{t}, \quad \vec{v}=\left(\vec{B}^{*}, \vec{H}^{*}\right)^{t}, \quad \vec{p}=(\lambda, \phi)^{t}$. The details of the saddle-point formulation can be found in [29].

We recall here the fact that the Schur complement was not defined for the symbol of the differential operator we associated with the saddle-point problem (4.9). This led us to perturb the operator and use the resulting Schur complement as a preconditioner. The Schur complement preconditioner (4.12) is scaled by the perturbation parameter $\epsilon$. One expects that the smaller the perturbation, the better the preconditioner. This is an interesting example where the 'optimal' limit $\epsilon=0$ leads to a singular preconditioner.

We solved the problem on $\Omega=[0,1]^{2}$ with both mixed and non-mixed boundary conditions. We used preconditioner (4.13), which corresponds to the perturbed operator $\mathcal{L}_{\epsilon}$ described in Section 4.4; in particular, the approximation to the Schur complement was assembled on a dual mesh as described in Section 5.1. The results for various perturbation parameters are displayed in Tables 5,6 . We see indeed that the smaller the perturbation parameter, the better the performance of GMRES, as we intuited from the form of the symbol (4.12) of the Schur complement. Note that we could not decrease the parameter $\epsilon$ any further without deterioration in performance. This is possibly due to rounding error.

Finally, we refer the reader to [30], [31] for Schur complement preconditioners of algebraic type as well as other preconditioning approaches. 


\begin{tabular}{l|cccc}
$h=$ & $1 / 16$ & $1 / 32$ & $1 / 64$ & $1 / 128$ \\
\hline$\epsilon=1$ & 36 & 33 & 33 & 33 \\
$1 e^{-3}$ & 30 & 28 & 28 & 28 \\
$1 e^{-6}$ & 27 & 24 & 25 & 26
\end{tabular}

Table 5: GMRES iteration counts for $\Gamma_{H}=\emptyset$.

\begin{tabular}{l|cccc}
$h=$ & $1 / 16$ & $1 / 32$ & $1 / 64$ & $1 / 128$ \\
\hline$\epsilon=1$ & 37 & 37 & 34 & 34 \\
$1 e^{-3}$ & 32 & 32 & 29 & 30 \\
$1 e^{-6}$ & 27 & 28 & 27 & 27
\end{tabular}

Table 6: GMRES iteration counts for $\Gamma_{H} \neq \emptyset$.

\section{Conclusion}

In this work we examined the issue of preconditioner design for elliptic regular systems. In particular, we proposed a strategy based on factorizing the symbol of the matrix differential operator, which in turn led to preconditioners based on the Schur complement of the symbol. Although in almost all our examples the Schur complement operator was a classical differential operator, this is not the case in general. The Oseen operator was an instructive, yet practical example to consider where the Schur complement was a pseudodifferential operator. The choice of boundary conditions usually depends on both preconditioned and preconditioning operators. The work of Chen and Strikwerda who pointed out the relation between operators and good preconditioners based on the Douglis-Nirenberg numbers was useful in establishing the ellipticity and thus suitability of the Schur complement preconditioners we suggested; moreover, their guidelines helped define suitable boundary conditions for our preconditioning operators. The resulting solvers exhibit no mesh dependence and fast convergence. However, the analysis of preconditioned iterations is available case by case. This highlights the need for a general analysis of preconditioned finite element discretizations of regular elliptic systems which preserve the regularity of the problem. We note here that some results are available for finite difference discretizations [9].

Finally, we note that some of the preconditioners considered in the previous section have already been suggested in the literature, one of the reasons being that the symbols of the operators involved are not too complicated. This allowed many practitioners to derive useful preconditioners using a purely algebraic approach. However, we expect our methodology to provide answers in the case of more complicated and larger matrix operators than the ones considered.

\section{References}

[1] S. Agmon, A. Douglis, and L. Nirenberg. Estimates near the boundary for solutions of elliptic partial differential equations satisfying general boundary conditions II. 
Comm. Pure Appl. Math., 17:35-92, 1964.

[2] D. N. Arnold, R. S. Falk, and R. Winther. Preconditioning discrete approximations of the Reissner-Mindlin plate model. RAIRO Model. Math. Anal. Numer., 31(4):517-557, 1997.

[3] I. Babuska, J. T. Oden, and J. K. Lee. Mixed-hybrid finite element approximations of second-order elliptic boundary-value problems, Part 1. Comput. Meth. Appl. Mech. Engrg, 11:175-206, 1977.

[4] I. Babuska, J. T. Oden, and J. K. Lee. Mixed-hybrid finite element approximations of second-order elliptic boundary-value problems, Part 2: Weak-hybrid methods. Comput. Meth. Appl. Mech. Engrg, 14:1-22, 1978.

[5] J. H. Bramble and J. E. Pasciak. A preconditioning technique for indefinite systems resulting from mixed approximations of elliptic problems. Math. Comp., 50:1-17, 1988.

[6] F. Brezzi and M. Fortin. Mixed and hybrid finite element methods. Springer-Verlag, New York, 1991.

[7] J. Cahouet and J. P. Chabard. Some fast 3D finite element solvers for the generalized Stokes problem. Internat. J. Numer. Methods Fluids, 8(8):869-895, 1988.

[8] Z. Cai, R. Lazarov, T. Manteuffel, and S. McCormick. First-oder system least squares for second-oder partial differential equations. SIAM J. Num. Anal., 31:1785-1799, 1994.

[9] H. H. Chen and J. C. Strikwerda. Preconditioning for regular elliptic systems. SIAM J. Num. Anal., 37(1):131-151, 1999.

[10] Z. Chen, R. E. Ewing, and R. Lazarov. Domain decomposition algorithms for mixed methods for second order elliptic problems. Math. Comp., 65:467-490, 1996.

[11] A. Douglis and L. Nirenberg. Interior estimates for elliptic systems of partial differential equations. Comm. Pure Appl. Math., 8:503-538, 1955.

[12] H. C. Elman and D. J. Silvester. Fast nonsymmetric iterations and preconditioning for Navier-Stokes equations. SIAM J. Sci. Comp., 17:33-46, 1996.

[13] H. C. Elman, D. J. Silvester, and A. J. Wathen. Performance and analysis of saddle point preconditioners for the discrete steady-state Navier-Stokes equations. Technical Report UMCP-CSD:CS-TR-4164, Department of Computer Science, Maryland, 2000.

[14] G. I. Eskin. Boundary Value Problems for Elliptic Pseudodifferential Equations. AMS, 1981. 
[15] V. Faber, T. A. Manteuffel, and S. V. Parter. On the theory of equivalent operators and application to the numerical solution of uniformly elliptic partial differential equations. Adv. Appl. Math., 11:109-163, 1990.

[16] B. Fischer, A. J. Wathen, and D. J. Silvester. The convergence of iterative solution methods for symmetric and indefinite linear systems. In Numerical analysis 1997 (Dundee), pages 230-243. Longman, Harlow, 1998.

[17] R. Glowinski and M. Wheeler. Domain decompostion and mixed finite element methods for elliptic problems. In R. Glowinski et. al., editor, Frist International Symposium on Domain Decomposition Methods for Partial Differential Equations, pages 144-172. SIAM, Philadelphia, 1988.

[18] L. Hörmander. The Analysis of Linear Partial Differential Operators III: PseudoDifferential Operators. Springer-Verlag, 1985.

[19] I. C. F. Ipsen. A note on preconditioning non-symmetric matrices. SIAM J. Sci. Comput., to appear.

[20] D. Kay and D. Loghin. A Green's function preconditioner for the steady-state Navier-Stokes equations. Technical Report 99/06, Oxford University Computing Laboratory, 1999.

[21] D. Kay, D. Loghin, and A. J. Wathen. A preconditioner for the Steady-State Navier-Stokes equations. SIAM J. Sci. Comput., submitted 2000.

[22] A. Klawonn. Block-triangular preconditioners for saddle-point problems with a penalty term. SIAM J. Sci. Comput., 19(1):172-184, 1998.

[23] D. Loghin. Green's Functions for Preconditioning. PhD thesis, Oxford University, 1999.

[24] D. Loghin. Analysis of preconditioned Picard iterations for the Navier-Stokes equations. Submitted to Numer. Math, 2001.

[25] T. A. Manteuffel and S. V. Parter. Preconditioning and boundary conditions. SIAM J. Num. Anal., 27:656-694, 1989.

[26] M. F. Murphy, G. H. Golub, and A. J. Wathen. A note on preconditioning for indefinite linear systems. SIAM J. Sci. Comp., 21:1969-1972, 2000.

[27] J.-C. Nedelec. Mixed finite element in $\mathbf{r}^{3}$. Numer. Math., 35(315-341), 1980.

[28] A. I. Pehlivanov, G. F. Carey, and R. D. Lazarov. Least-squares mixed finite element for second-order elliptic problems. SIAM J. Num. Anal., 31:1368-1377, 1994.

[29] I. Perugia. A field-based mixed formulation for the two-dimensional magnetostatic problem. SIAM J. Numer. Anal., 34:2382-2391, 1997. 
[30] I. Perugia and V. Simoncini. Optimal and quasi-optimal preconditioners for certain mixed finite element approximations. Technical Report 1098, Istituto di Analisi Numerica del C.N.R., Pavia, 1998.

[31] I. Perugia, V. Simoncini, and M. Arioli. Linear algebra methods in a mixed approximation of magnetostatic problems. SIAM J. Sci. Comput., 21(3):1085-1101, 1999.

[32] P.-A. Raviart and J.-M. Thomas. A mixed finite element method for second order elliptic problems. In I. Galligani and E. Magenes, editors, Mathematical Aspects of Finite Element Methods, number 606 in Lecture Notes in Mathematics, pages 292-315. Springer, Berlin, 1977.

[33] P.-A. Raviart and J.-M. Thomas. Dual finite element models for second order elliptic problems. In R. Glowinsky, E. Y. Rodin, and O. C. ZienKiewicz, editors, Energy Methods in Finite Element Analysis, pages 175-191. Wiley, Chichester, 1979.

[34] J. N. Reddy and J. T. Oden. Mixed finite element approximations of linear boundary-value problems. Quart. Appl. Math, 33(255-280), 1975.

[35] J. E. Roberts and J.-M. Thomas. Mixed and hybrid methods. In P. G. Ciarlet and J. L. Lions, editors, Hanbook of Numerical Analysis, volume 2, pages 523-641. Elsevier, 1991.

[36] T. Rusten and R. Winther. Substructure preconditioners for elliptic saddle point problems. Math. Comp., 60(201):23-48, 1993.

[37] D. J. Silvester and A. J. Wathen. Fast iterative solution of stabilised Stokes systems. II. Using general block preconditioners. SIAM J. Num. Anal., 31(5):1352-1367, 1994.

[38] M. Taylor. Pseudodifferential Operators. Princeton University Press, 1981.

[39] P. S. Vassilevski and J. Wang. Multilevel iterative methods for mixed finite element discretizations of elliptic problems. Numer. Math., 63:503-520, 1992.

[40] A. J. Wathen and D. J. Silvester. Fast iterative solution of stabilised Stokes systems. I. Using simple diagonal preconditioners. SIAM J. Num. Anal., 30(3):630-649, 1993.

[41] J. Wloka, B. Rowley, and B. Lawruk. Boundary Value Problems for Elliptic Systems. Cambridge University Press, New York, 1995. 